\title{
Interactive comment on "What could we learn about climate sensitivity from variability in the surface temperature record?" by James Douglas Annan et al.
}

\section{James Douglas Annan et al.}

jdannan@blueskiesresearch.org.uk

Received and published: 11 May 2020

Thank you for your interesting and helpful comments. We believe that although the relationship with Psi is as you note dimensionally inconsistent, this doesn't actually indicate any error as the missing dimensions are effectively included in the constant of proportionality through the implicit assumption of Cox et al that all other model parameters (some of which have dimensions attached) are held fixed. Relating to your comments about detrending, as the stated purpose was to remove the forced trend, we did not perform this step for the unforced simulations. However doing so does not significantly affect our results, a point that will be noted in the revision. 
The reason for the inclusion of epsilon in the model is simply that it is widely regarded as a significantly better model for the inclusion of this term. It has very little influence ESDD on our results (at least if we replaced gamma*epsilon by gamma in our simulations) as we do not examine deep ocean temperature. The simulations in which $S$ alone varies do provide an end case to the situation of excessive uncertainty in other parameters.

Interactive comment

Interactive comment on Earth Syst. Dynam. Discuss., https://doi.org/10.5194/esd-2019-90, 2020. 